\title{
Valproic acid-induced DRESS in a child responding to cyclosporine with HLA analysis
}

\author{
Nazli Ercan $\mathbb{D}^{\mathbb{D}}$, Cengiz Zeybek $^{b}(\mathbb{D})$, Ayşe I. Atasoyc (D), Bülent Unay ${ }^{d}(\mathbb{D}$
}

\begin{abstract}
Drug reaction with eosinophilia and systemic symptoms (DRESS), also known as drug-induced hypersensitivity syndrome, is a potentially life-threatening rare reaction that causes a severe rash and can lead to multiorgan failure. As in other severe drug eruptions, drug-specific T lymphocytes play a crucial role in DRESS. The hapten/pro-hapten model, pharmacological interaction model, and altered peptide repertoire model are three different models developed to describe the relationship/interaction between a medication or its metabolites and the immune system. We discuss our experience with cyclosporine treatment in a steroid-resistant DRESS syndrome caused by valproic acid in a girl, as well as her clinical, laboratory, and human leukocyte antigens (HLA) study results.

Key words: child, cyclosporine, drug hypersensitivity syndrome, HLA antigens, valproic acid.
\end{abstract}

http: / / dx.doi.org/10.5546/ aap.2022.eng.e80

To cite: Ercan N, Zeybek C, Atasoy AI, Unay B. Valproic acid-induced DRESS in a child responding to cyclosporine with HLA analysis. Arch Argent Pediatr 2022;120(2):e80-e84.

a. Department of Child Health and Diseases, Division of Pediatric Allergy and Immunology, University of Health Sciences Turkey, Gülhane Training and Research Hospital, Ankara, Turkey.

b. Department of Child Health and Diseases, Division of Pediatric Nephrology, University of Health Sciences Turkey, Gülhane Training and Research Hospital, Ankara, Turkey.

c. Department of Child Health and Diseases, University of Health Sciences Turkey, Gülhane Training and Research Hospital, Ankara, Turkey.

d. Department of Child Health and Diseases, Division of Pediatric Neurology, University of Health Sciences Turkey, Gülhane Training and Research Hospital, Department of Child Health and Diseases, Ankara, Turkey.

E-mail address:

Nazli Ercan: drnazliercan@gmail.com

Funding: None.

Conflict of interest: None.

Received: 3-6-2021

Accepted: 9-1-2021

\section{INTRODUCTION}

DRESS syndrome (drug reaction with eosinophilia and systemic symptoms) is a rare drug-induced hypersensitivity reaction that causes a severe rash and can progress to multiorgan failure and death. It may occur within the first 3-12 weeks of initiation of a new medication. ${ }^{1}$ DRESS syndrome is thought to be caused by a unique interaction of the causative drug with $\mathrm{T}$ lymphocytes, which leads to herpesvirus reactivation, which is aided by the individual's immunogenetic susceptibility, particularly the major histocompatibility complex (HLA). ${ }^{2}$ Anticonvulsants, antibiotics, and allopurinol are usually blamed for the disease's etiology, with a fatality rate of about $10 \% .^{1}$ The specific diagnosis is based upon the history, clinical, and biochemical parameters of the patient. The standard treatment regimen is to immediately discontinue the suspected drug and administer systemic corticosteroids with supportive treatment if there is organ involvement. ${ }^{1}$ Immunoglobulin, cyclophosphamide, mycophenolate mofetil, rituximab, and cyclosporine. are alternative therapies with limited data in adults. We report our experience in the treatment of a girl with valproic acid (VPA)-associated steroid-resistant DRESS syndrome with cyclosporine, along with haplotype analysis.

\section{CASE REPORT}

A 15-year-old girl of Caucasian race was administered oral methylprednisolone (3 days) and cetirizine (1 week) for itchy maculopapular eruptions, that started 15 days before on her legs and arms, and then spread to the trunk. She was consulted to the pediatric allergy clinic due to persistent fever, swelling in the auricles and around the eyes, and a rash that spread to the whole body. She had been receiving risperidone treatment for a year because of bipolar disorder, and VPA was added to the treatment for the last three weeks. At her initial physical examination, the patient's general condition was moderate, conscious, body temperature: $38.5^{\circ} \mathrm{C}$, respiratory rate: $24 / \mathrm{min}$, heart rate: $85 / \mathrm{min}$, and blood 
pressure: $90 / 60 \mathrm{mmHg}$. The patient had edema around the eyes, auricle, and face, diffuse erythema on the face and neck, and generalized rash in the morbilliform eruptions (Figure 1). Nikolsky's sign was negative. Cracks in the mouth's rim, oropharyngeal hyperemia, multiple lymphadenopathies of $1 \times 1 \mathrm{~cm}$ in the right and left anterior cervical chain were detected. Dullness to percussion over Traubess space was shown as splenomegaly. She was hospitalized with a diagnosis of drug allergy. Laboratory analysis

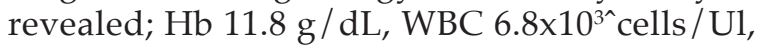
Plt $145 \times 10^{3}$ cells / Ul with prominent eosinophilia in peripheral smear with atypical lymphocytes (Figure 2). Liver (LFTs) and renal function tests (RFTs) were within normal limits. C-reactive protein (CRP) was $66.10 \mathrm{mg} / \mathrm{L}$ (Figure 3). Total protein and albumin were low at $4.99 \mathrm{~g} / \mathrm{dL}$ and $1.86 \mathrm{~g} / \mathrm{dL}$, respectively. There was significant proteinuria $(+3)$ in urine analysis. Blood, urine cultures, respiratory multiplex PCR (including SARS-CoV-2), and autoimmune disease screening tests were negative. Chest $\mathrm{x}$-ray and echocardiography were within normal limits. The Registry of Severe Cutaneous Adverse Reactions (RegiSCAR) score of 9 was suggestive of DRESS syndrome associated with VPA; thus, VPA was discontinued immediately.

Treatment was started with methylprednisolone $(1 \mathrm{mg} / \mathrm{kg} / 24 \mathrm{hr} \div \mathrm{Q} 12 \mathrm{hr})$, pheniramine hydrogen maleate IV, and as a supportive therapy topical clobetasol propionate for 5 days. The patient still had a persistent fever $\left(40.1^{\circ} \mathrm{C}\right)$ on the 4 th day, and the CRP was $124.86 \mathrm{mg} / \mathrm{L}$, urea $450 \mathrm{mg} / \mathrm{L}$, and creatinine $17 \mathrm{mg} / \mathrm{L}$ (Figure 3, 4). Therefore, we switched to pulse methylprednisolone (3 days IV $1000 \mathrm{mg} /$ day) and intravenous immunoglobulin $1 \mathrm{~g} / \mathrm{kg} /$ day for 4 days. On the second day of pulse steroid therapy, the patient developed a dry cough, tachypnea, and hypoxia, and increased fever $\left(39.4^{\circ} \mathrm{C}\right)$ (Figure 4). SARS-CoV-2 nasopharyngeal swab testing was negative. Diffuse infiltration was present on chest X-ray, and pulmonary highresolution computed tomography showed diffuse nodular ground-glass appearance suggesting atypical pneumonia. Induced sputum culture for Pneumocystis Jiroveci (PJ) was positive. Concurrently, CRP decreased, but RFTs and LFTs increased. Cyclosporine $100 \mathrm{mg}$ orally two times a day for 3 days, meropenem IV (10 days), trimethoprim-sulfamethoxazole p.o. (14 days) and clarithromycin IV (7 days) was added to the steroid regimen. Her symptoms

FIGURE 1. a. Edema around the eyes, auricle, and face, diffuse erythema on her face and neck, $\boldsymbol{b}$ : Morbiliform rash on the trunk

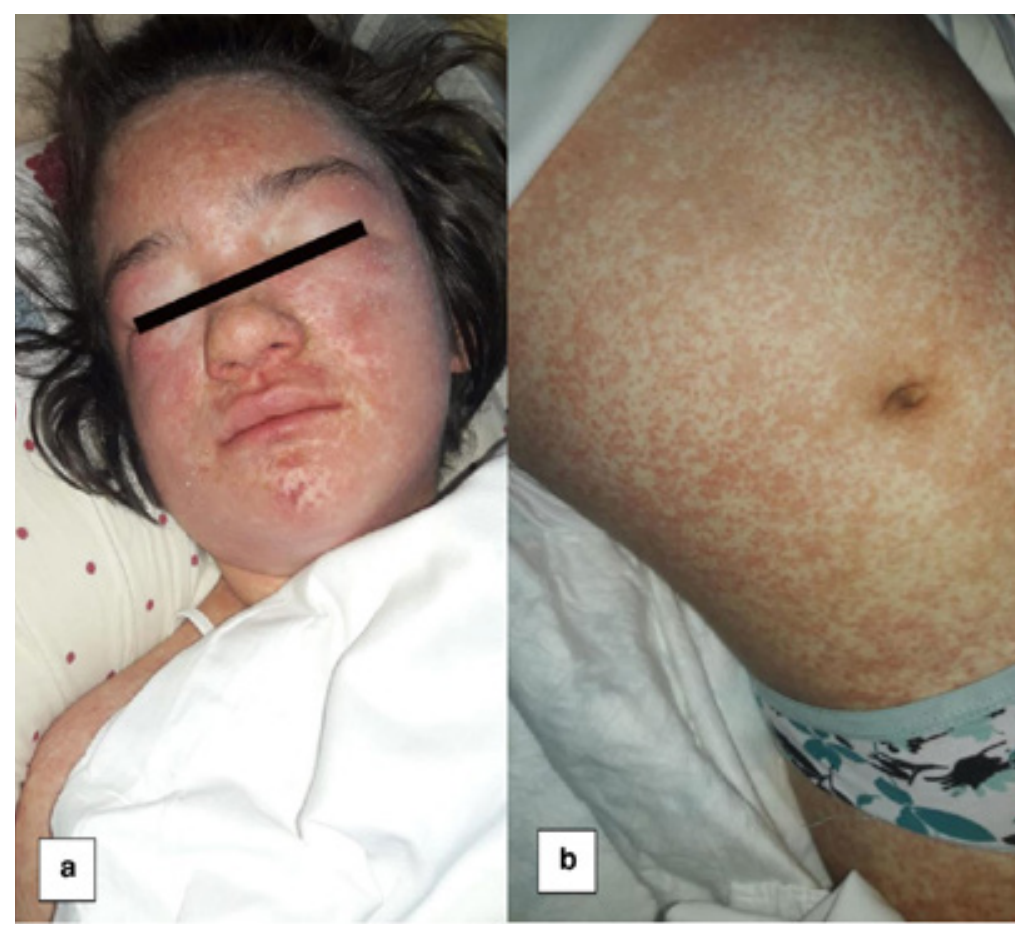


improved, and RFTs regressed after 24-48 hours. DRESS syndrome flare-up occurred one week after discharge, accompanied by human herpesvirus type 6 reactivation. Liver function tests improved three days after adding oral cyclosporine $2 \times 100 \mathrm{mg}$ three times a week to methylprednisolone and TMP-SMX treatment.

Oral cyclosporine and methylprednisolone

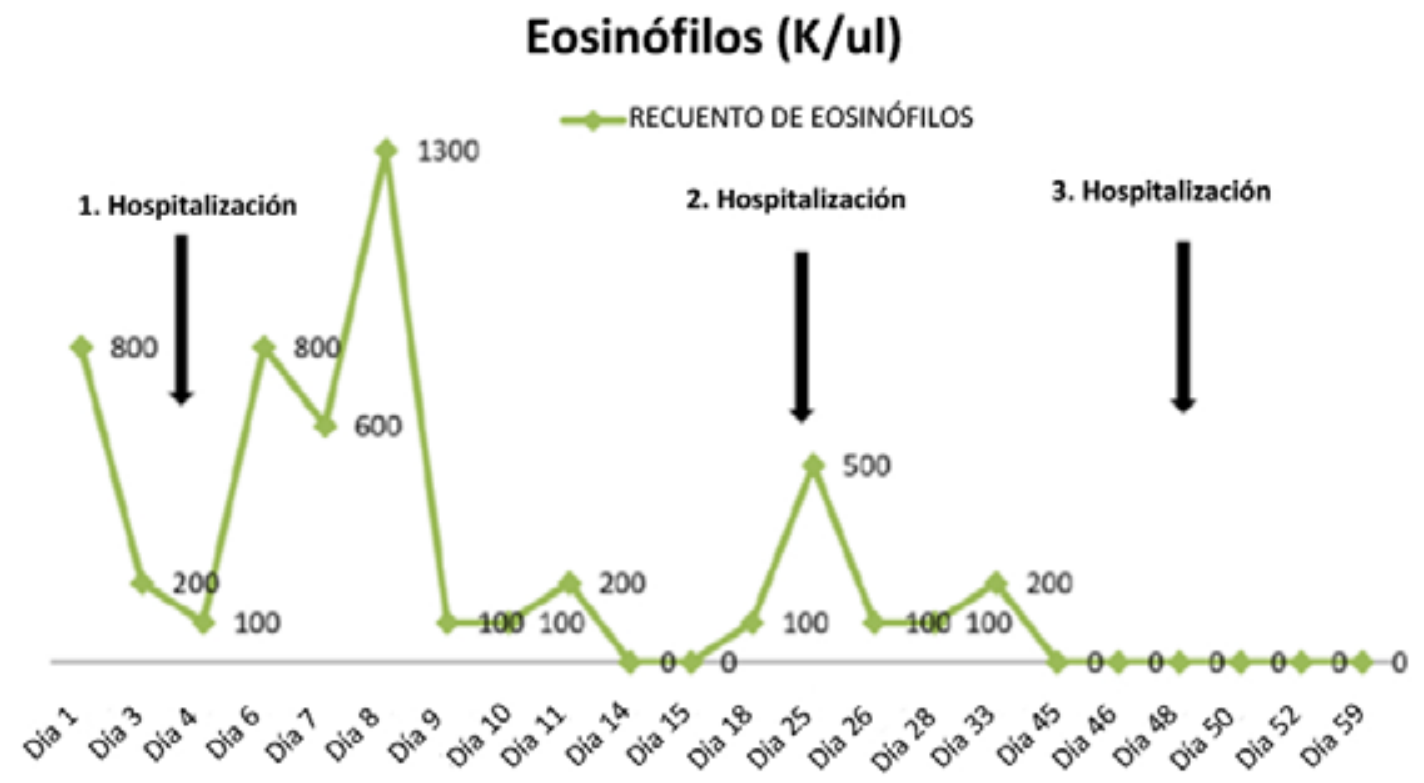

FIGURE 3. The monitorization of CRP, urea, creatinine levels of the patient

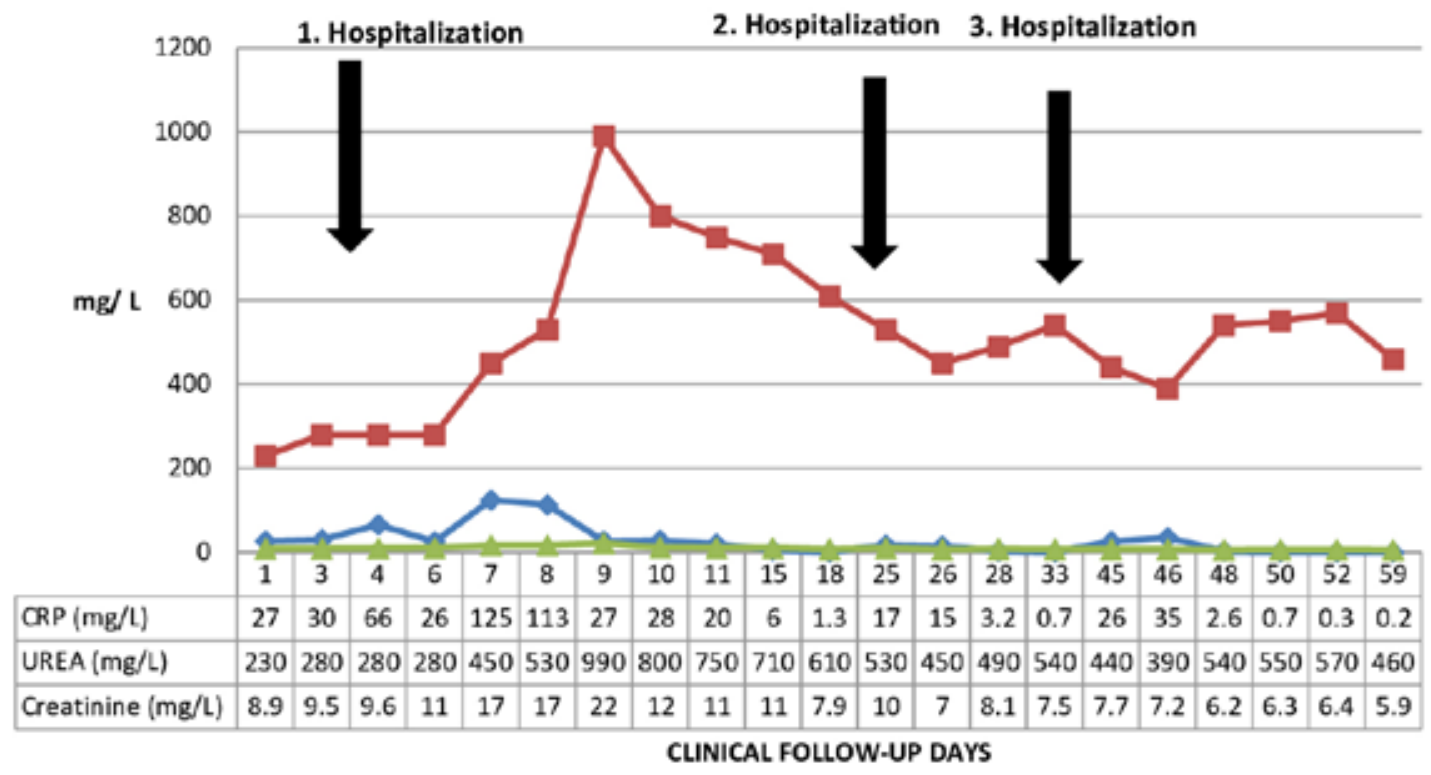


were continued with a slow taper over 24 months due to relapse of rash, RFTs, and LFTs deterioration at 3, 6, and 12 months after hospitalization. Steroid-induced avascular necrosis and DRESS-induced tubulointerstitial nephritis were detected in her follow-up. Patch testing with 20\% VPA after complete regression of DRESS (on the 6th month) showed type IV sensitization to VPA. This result supports the cause-effect relationship of VPA and DRESS. The following is the haplotype analysis we performed on our patient, which can be utilized to predict the onset of DRESS caused by VPA: HLA-A*24:30, HLA-B*49: 53, HLA-C*07:04, HLA-DRB1*07:04, and HLA-DQB1*02:06.

The patient continued to use risperidone for bipolar disorder without experiencing any adverse reactions. No autoimmune diseases or cancer was detected throughout our patient's two years follow-up.

\section{DISCUSSION}

DRESS syndrome is fatal in about $10 \%$ of patients, with liver necrosis being the most prevalent cause. ${ }^{1}$ The clinical symptoms of DRESS (edema, rash, lymphadenopathy, fever) as well as indicators of hematologic or internal organ involvement in laboratory data are used to diagnose the syndrome. ${ }^{1}$ The treatment for DRESS syndrome includes both a prompt withdrawal of the probable causal agent along with supportive care. The steroid is frequently the first choice as a systemic immunosuppressant in patients with internal organ involvement. This case study is novel in demonstrating cyclosporine as a possible alternative treatment option after step-line treatment in DRESS unresponsive to steroids at pediatric age. In a small number of adult patients with DRESS, cyclosporine has been used in first or second-line therapy, in cases of steroid unresponsiveness or side effects or relapse of DRESS. ${ }^{1,3-8}$ Nguyen et al., employed cyclosporine as a first-line treatment in 5 adult patients. Despite the initial improvement, two patients discontinued therapy from adverse effects, which were reversible on discontinuation. ${ }^{8}$ Although we preferred a pulse steroid regimen in our second step treatment, the benefit is controversial, as reported in one case report. ${ }^{4}$ In our case, pulse steroid therapy might have triggered the steroid-dependent DRESS variant by causing HHV-6 viral reactivation secondary to immune suppression. ${ }^{1}$

The generation of interleukin 5 by $\mathrm{T}$ cells, which leads to eosinophilia, could be a key factor in the pathophysiology of drug hypersensitivity syndrome. T cells are inhibited in their production of interleukin 5 by corticosteroids and

\section{Fever $\left({ }^{\circ} \mathrm{C}\right) \quad$ 3.Hospitalization}
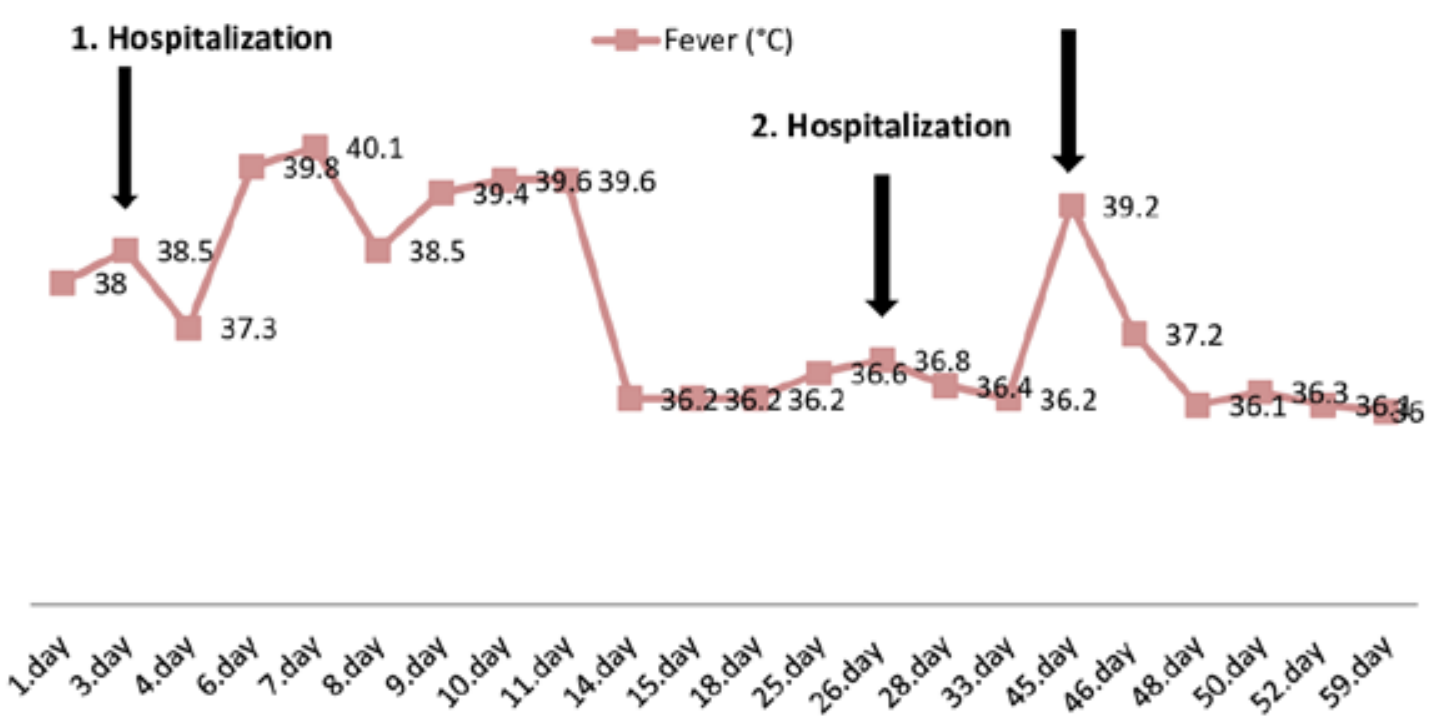
cyclosporine. ${ }^{5}$ Thus, $200 \mathrm{mg}$ of cyclosporine daily may be a viable alternative to corticosteroids in a steroid-resistant DRESS patient of adolescent age.

The pathophysiology of hypersensitivity is thought to entail a combination of poor pharmacokinetics and drug metabolite accumulation, herpesvirus reactivation in a sequential manner, and genetic predisposition imparted by connection with a particular HLA class I alleles. ${ }^{1}$ The immune system is thought to play a key role in drug hypersensitivity syndrome development. Certain HLA antigen haplotypes are known to predispose sufferers to drug hypersensitivity syndrome. HLA interact with drug metabolites before being delivered to $\mathrm{T}$ cells, causing $\mathrm{T}$ cells to become activated. ${ }^{5}$ Many ethnic groups in Europe and Asia have been discovered as having HLA, which has been linked to the development of severe adverse drug reactions, such as DRESS, as a result of the use of specific aromatic anticonvulsants (carmabezapine, lamatrigine, phenytoin). ${ }^{9}$ There is no HLA haplotype yet identified regarding the development of severe adverse drug reaction after the use of VPA, a non-aromatic anticonvulsant. To our knowledge, this is the first case reporting the HLA analysis in VPA-induced DRESS syndrome in children. In only one adult case, haplotype analysis for VPA has been published, in which maculopapular eruption was detected due to the use of multiple psychotropic drugs consisting of VPA, chlorpromazine, and carbamazepine. The haplotype analysis for the suspected agent (VPA) revealed HLA-A 2402, HLA-B4601, and HLA-A0207. ${ }^{10}$

Cyclosporine can be a promising alternative treatment among immunosuppressants in pediatric patients who are resistant or having side effects or relapse with steroids. In addition, HLA haplotype analysis will not only provide a foundation for future research but will also aid in the prediction of severe adverse drug reaction development, such as DRESS syndrome.

\section{REFERENCES}

1. Kirchhof MG, Wong A, DutzJP. Cyclosporine Treatment of Drug-Induced Hypersensitivity Syndrome.JAMA Dermatol. 2016; 152(11):1254-7.

2. Lefebvre M, Walencik A, Allavena C, Billaud E, et al. Rate of DRESS Syndrome With Raltegravir and Role of the HLA-B*53: 01 Allele. J Acquir Immune Defic Syndr. 2020; 85(4):e77-80.

3. Harman KE, Morris SD, Higgins EM. Persistent anticonvulsant hypersensitivity syndrome responding to ciclosporin. Clin Exp Dermatol. 2003; 28(4):364-5.

4. Lee JH, Park HK, Heo J, Kim TO, et al. Drug Rash with Eosinophilia and Systemic Symptoms (DRESS) syndrome induced by celecoxib and anti-tuberculosis drugs. J Korean Med Sci. 2008; 23(3):521-5.

5. Zhang ZX, Yang BQ, Yang Q, Wu M, Wang GJ. Treatment of drug-induced hypersensitivity syndrome with cyclosporine. Indian J Dermatol Venereol Leprol. 2017; 83(6):713-7.

6. Hashizume H, Kageyama R, Kaneko Y. Short course of cyclosporin A as a treatment option for drug-induced hypersensitivity syndrome: Case reports and review of the published work. J Dermatol. 2018; 45(6):e169-70.

7. Ton A, Kassab L, Patel A, Dawson N. Severe acute hepatitis in drug reaction with eosinophilia and systemic symptoms (DRESS) syndrome resolved following cyclosporine. J Allergy Clin Immunol Pract. 2020; 8(1):398-400.

8. Nguyen E, YanesD, ImadojemuS, Kroshinsky D. Evaluation of Cyclosporine for the Treatment of DRESS Syndrome. JAMA Dermatol. 2020; 156(6):704-6.

9. Martínez-Cabriales SA, Rodríguez-Bolaños F, Shear NH. Drug Reaction with Eosinophilia and Systemic Symptoms (DReSS): How Far Have We Come? Am J Clin Dermatol. 2019; 20(2):217-36.

10. Sakurada K, Kozaru T, Yamada K, Nibuya M, etal. Allergy to chlorpromazine and valproic acid following carbamazepine hypersensitivity in a patient with an HLA-B ${ }^{\star} 4601$ allele. Neuropsychiatr Dis Treat. 2018; 14:1139-42. 